Published in final edited form as:

AIDS Behav. 2018 September ; 22(9): 3003-3008. doi:10.1007/s10461-018-2094-5.

\title{
Travel time to Clinic but not Neighborhood Crime Rate is associated with Retention in Care among HIV-positive Patients
}

\author{
Jessica P. Ridgway ${ }^{1,2}$, Ellen A. Almirol ${ }^{2}$, Jessica Schmitt ${ }^{1,2}$, Todd Schuble ${ }^{3}$, and John A. \\ Schneider ${ }^{1,2,4, \S}$ \\ ${ }^{1}$ Department of Medicine, University of Chicago, Chicago, IL, USA \\ ${ }^{2}$ Chicago Center for HIV Elimination, University of Chicago, Chicago, IL, USA \\ ${ }^{3}$ Research Computing Center, University of Chicago, Chicago, IL, USA \\ ${ }^{4}$ Department of Public Health Sciences, University of Chicago, Chicago, IL, USA
}

\section{Abstract}

Using geospatial analysis, we examined the relationship of distance between a patient's residence and clinic, travel time to clinic, and neighborhood violent crime rates with retention in care or viral suppression among people living with HIV (PLWH). For HIV-positive patients at a large urban clinic, we measured distance and travel time between home and clinic and violent crime rate within a two block radius of the travel route. Kruskal-Wallis rank sum was used to compare outcomes between groups. Over the observation period, 2008-2016, 219/602 (36\%) patients were retained in care. Median distance from clinic was 3.6 [IQR 2.1-5.6] miles vs. 3.9 [IQR 2.7-6.1] miles among those retained vs. not retained in care, $\mathrm{p}=0.06$. Median travel time by car was 15.9 [IQR 9.6-22.9] vs. 17.1 [IQR 12.0-24.6] minutes for those retained vs. not retained, $\mathrm{p}=0.04$. Violent crime rate along travel route was not associated with retention. There was no significant association between travel time or distance and viral suppression.

\section{Keywords}

Retention in care; HIV care continuum; geospatial analysis

\section{Background}

Local geographic and contextual factors can have a significant impact on the health of people living with HIV (PLWH). Within a given city, HIV care continuum outcomes, such as linkage to care (LTC) and retention in care (RIC), vary by neighborhood. For example, not only are diagnoses of HIV more common in specific neighborhoods(1), but individual

Corresponding author: John Schneider, MD, MPH, 5841 S Maryland Ave, MC 5065, Chicago, IL, 60637. Ph: 773-702-2710. Fax: 773-702-8998, jschnei1@ medicine.bsd.uchicago.edu.

Conflict of Interest: JR and JS have received grants from Gilead Sciences. The other authors report no conflicts of interest.

Ethical approval: This study was approved by the University of Chicago Institutional Review Board. All procedures performed in studies involving human participants were in accordance with the ethical standards of the institutional and/or national research committee and with the 1964 Helsinki declaration and its later amendments or comparable ethical standards. For this type of study formal consent is not required. 
PLWH with poor RIC also tend to reside in geographic clusters of poor RIC $(2,3)$. These local geographic variations in HIV care continuum metrics may be explained by physical and structural barriers to care (e.g., lack of access to healthcare or transportation) (4) or by sociocontextual factors that can affect engagement in care, such as such as poverty or crime (5).

Geographic information systems (GIS) technology provides the ability to analyze spatial risk factors for poor engagement in care. While GIS technology has been used to map community-level patterns of HIV care continuum metrics $(2,3,6)$, we are unaware of previous work using geospatial analysis to evaluate the impact of spatial risk factors on engagement in care and viral suppression at the individual level. Using geospatial analysis, we aimed to determine if distance between a patient's residence and HIV care clinic, travel time to clinic, or violent crime rate along travel route to clinic are associated with retention in care or viral suppression (VS) among PLWH.

\section{Methods}

\section{Data collection and geocoding}

We abstracted demographics, laboratory data, address, and medical visit history for all patients $\geq 18$ years old who received primary HIV care at our urban clinic from January 1, 2008 through May 10, 2016. Our HIV care clinic is a major provider of HIV care on the south side of Chicago, one of the major domestic HIV epicenters.

Patients' home addresses and the location of the clinic were geocoded using ESRI's StreetMap Premium geocoding service (7). The geocoding service produced an XY coordinate pair for each street address, which were then mapped. Using the patient's home address as the origin and the clinic as the destination, three separate network calculations were performed. The first calculation determined a patient's travel distance to clinic along a street network in a private vehicle. The second calculation determined a patient's travel time to clinic along a street network in a private vehicle. The third calculation determined a patient's travel time to clinic using only walking and the Chicago Transit Authority (CTA) buses and trains as modes of transportation $(8,9)$.

The first two network calculations used street network data provided by ESRI's StreetMap Premium service. Using ESRI's ArcGIS Network Analyst module, the shortest route using distance and time as separate cost measures produced a length product in miles and a time product in minutes for each starting and ending point. The third calculation used street centerline data from the City of Chicago's Data Portal (8). The data were modified to display only pedestrian right-of-ways. In addition, CTA route schedules (9) were used to create a connectivity network navigable by pedestrians. By creating a specialized network using ESRI's Network Analyst module (7) that would optimize the CTA schedule, a route was produced for each patient to reach the HIV care clinic using time as a cost.

One further calculation produced the crime rate within a two block radius of a patient's route to clinic if they utilized the CTA to and from their destination. XY coordinates for violent crime data from 2012 to 2016 were provided by the City of Chicago's Data Portal (8). The 
crime rates were normalized using block group demographics from the US Census Bureau (10). Average crime rates within two blocks of each individual patient route were then calculated. While crime rates were not calculated for the entire study period, crime rates within the study area fluctuate yearly by $5 \%$ or less. Therefore, it was deemed unnecessary to collect more intricate historical crime data. Patients who lived outside of Chicago city limits were excluded from the geospatial analyses because we did not have access to accurate crime data or transit schedule data for areas outside of the city of Chicago.

\section{Definitions and statistical analyses}

Retention in care was defined as $\geq 2$ visits with a HIV care provider, $\geq 90$ days apart within a 12-month period (11). While many RIC metrics exist, this definition has been shown to be correlated with viral suppression and improved patient outcomes (12). Patients were classified as continuously retained in care if they met this definition for every 12-month period starting from the date of their first HIV care visit. Viral suppression (VS) was defined as viral load 200 copies/ml at the most recent visit. Individuals were censored if patients moved out of Chicago, transferred care to another provider, or deceased. Our clinic routinely monitors patients' visit adherence and has several methods to retain patients in care, including reminder phone calls/text messages prior to appointments and rescheduling after missed visits. For patients who have not been seen in clinic in over 6 months, we attempt to contact patients and their emergency contacts telephonically, send a certified letter, and if necessary, perform a home outreach visit. Kruskal-Wallis rank sum was used to compare outcomes between groups. All statistical analyses were conducted in R 2.14.0.

\section{Results}

In total, 771 patients received primary HIV care at our clinic during the study time period. Of these, 602 lived within the city of Chicago and had a physical address recorded in the medical record (i.e. not a post office box). The majority of patients were male $(60.0 \%)$, African American (86.5\%), and had Medicaid or Medicare insurance (61.8\%). 219/602 (36.4\%) patients were continuously retained in care. Table I shows demographics by retention group and viral suppression. Patients who were not virally suppressed were more likely to be younger than 25 years of age, have Medicaid insurance, and be nonwhite than those who were virally suppressed $(9.5 \%$ vs. $3.2 \%, \mathrm{p}=0.03 ; 52.4 \%$ vs. $37.8 \%$; $\mathrm{p}=0.003$; $96.8 \%$ vs. $91.1 \%, \mathrm{p}=0.03$, respectively). Among those who were continuously retained, $85.7 \%$ were virally suppressed. Average length of clinic attendance was 4.4 years over the study period.

The majority of patients, $52.0 \%$ (313/602), lived within a 4 mile radius of our clinic. Figure 1 displays maps of geocoded data, including patients' residence locations, travel time, and violent crime rate along travel route from home to clinic. Table II shows travel distance, travel time by car and CTA, and violent crime rate along the public transportation route, stratified by retention and viral suppression status. Shorter travel time by car was significantly associated with retention in care (15.9, IQR [9.6-22.9], minutes for clients retained in care vs. 17.1, IQR [12.0-24.6] minutes for clients not retained in care, $\mathrm{p}=0.04$ ). Although not reaching statistical significance, there were also trends toward negative 
associations between travel distance and retention in care (3.6 miles for clients RIC vs. 3.9 miles for clients not RIC, $\mathrm{p}=0.06)$ and travel time by CTA and retention in care (37.2 minutes for clients RIC vs. 42.9 for clients not RIC, $\mathrm{p}=0.07$ ). There was no difference in violent crime rate along the travel route between clients RIC vs. clients not RIC. Distance and travel time from the patients' home to clinic were not associated with viral suppression, but a trend towards lower violent crime rates along the travel route for clients who were virally suppressed vs. those not virally suppressed was observed ( 0.12 violent crimes/ $100,000$ population vs. 0.13 violent crimes $/ 100,000$ population, $\mathrm{p}=0.07)$.

\section{Discussion}

In our study of PLWH who receive care at a large HIV clinic on the south side of Chicago, we found that individuals with a longer travel time between home and clinic were less likely to be retained in care. Interestingly, we found no significant association between travel time to clinic and viral suppression. Although longer travel time likely impacted patients' likelihood of attending medical visits for HIV, adherence to anti-retroviral therapy was not affected. While retention in care is usually highly correlated with viral suppression (13), care plans vary by patient with providers prescribing long courses of antiretroviral therapy (e.g., one-year supply) or prescribing refills over the phone with patients who are known or have a history of maintaining viral suppression. Patients who live far away from their HIV provider might find it difficult to attend frequent medical visits, but still find ways to access to antiretroviral therapy to achieve viral suppression. Nevertheless, retention in care is important for the health of PLWH independent of viral suppression $(14,15)$, and so our findings suggest it is important to find ways to facilitate access to care for those who live farther away from their HIV care providers.

Our findings differ from results of prior studies. Eberhart et al. found no association between distance to the nearest medical provider and retention in care (2). However, this study examined only the distance between the client's home and the nearest medical provider; they did not measure distance from the patients' homes to their actual HIV care providers. Patients do not necessarily access care at the HIV care provider nearest to their home (16). Our study may be more accurate because we mapped distance and travel time between patients' homes and their specific HIV care provider.

Although longer travel time was associated with lower rates of retention in care, violent crime rate along the travel route was not associated with retention. We hypothesized that clients whose travel route to clinic traversed high-crime neighborhoods might be less likely to attend their HIV care appointments. Our data, however, did not show this to be true. Of note, clients with higher violent crime rate on their travel route did show a trend toward being less likely to be virally suppressed. This finding is consistent with prior literature suggesting that individuals who live in high-crime areas are more likely to have worse health outcomes or engage in unhealthy behaviors $(17,18)$. We also found that patients who were not virally suppressed were more likely to be younger or have Medicaid insurance (possibly indicating lower income), and be nonwhite. These findings are consistent with prior literature regarding risk factors for virologic failure (19). 
Our findings have several implications for HIV patient care. When linking newly diagnosed clients to care, besides client insurance status and provider preference, distance between a client's home and the clinic is important to consider. Even more than distance, travel time to clinic was an important predictor of retention in care. For clients who require assistance with transportation, clinics could consider providing a voucher for a vehicle ride share instead of bus passes, as travel time by car can be considerably shorter than by public transportation. Clinics should collaborate with patients to schedule appointments at convenient times for patients' schedule and travel plans (e.g., scheduling appointments with multiple providers on the same day, or avoiding scheduling during rush hour). Empowering patients to play more of a role in scheduling their appointments (e.g., through patient portals, etc.) could improve visit attendance $(20,21)$. Finally, since there was no significant difference in viral suppression based on travel time, it may be acceptable to schedule less frequent visits for clinically stable patients with longer travel time.

Our study has several notable strengths. Prior studies examining the impact of distance to clinic on retention in care relied on public health data, often using laboratory data as a proxy for medical visits $(2,3,16)$. However, we were able to measure patients' actual attendance at medical appointments. Similarly, while prior studies measured distance from a patient's residence to the closest medical provider, we measured distance and time to the client's actual HIV care provider $(2,3,16)$. Finally, our calculations are based on geocoding patient addresses, not on zip code or census tract data, providing an additional level of granularity and accuracy (6).

However, this study also has several limitations. First, we did not collect data regarding vehicle ownership among our patients. Therefore, we were not able to incorporate into our analyses clients' preferred route of transportation to clinic. Previous studies have shown that vehicle ownership is associated with improved linkage and viral suppression, independent of socioeconomic status (22). Furthermore, in our analyses we only considered clients' most recent address. It is possible that clients moved over time or were unstably housed. In addition, because this was a single center study, it is possible that patients may have been misclassified in their retention status. Some patients we classified as "not retained in care" may have transferred care without notifying our clinic. However, our clinic utilizes robust outreach efforts to contact and re-engage clients who are lost to follow-up, including telephonically, certified letters, and home visits. Finally, this study was performed in a predominantly African American, low socioeconomic status, urban population in Chicago. Results may not be generalizable to other populations and communities. Travel time and cost may vary depending on the city or community.

Local spatial risk factors and trends can have important implications for HIV care and public health as we seek to improve care across the HIV care continuum. Using geospatial analysis, we found that travel time by car but not crime rate was significantly associated with retention in care. Further research is needed regarding other geographic and neighborhood sociocontextual factors and their association with care continuum metrics. 


\section{Acknowledgments}

Funding: This study was funded by a grant from the NIH-funded Third Coast Center for AIDS Research (CFAR) (P30 AI117943).

\section{References}

1. Phillips G 2nd, Birkett M, Kuhns L, Hatchel T, Garofalo R, Mustanski B. Neighborhood-level associations with HIV infection among young men who have sex with men in Chicago. Arch Sex Behav. 2015; 44(7):1773-86. [PubMed: 26168977]

2. Eberhart MG, Yehia BR, Hillier A, Voytek CD, Blank MB, Frank I, et al. Behind the cascade: analyzing spatial patterns along the HIV care continuum. J Acquir Immune Defic Syndr. 2013; 64(Suppl 1):S42-51. [PubMed: 24126447]

3. Eberhart MG, Yehia BR, Hillier A, Voytek CD, Fiore DJ, Blank M, et al. Individual and community factors associated with geographic clusters of poor HIV care retention and poor viral suppression. J Acquir Immune Defic Syndr. 2015; 69(Suppl 1):S37-43. [PubMed: 25867777]

4. Mallinson RK, Relf MV, Dekker D, Dolan K, Darcy A, Ford A. Maintaining normalcy: a grounded theory of engaging in HIV-oriented primary medical care. ANS Adv Nurs Sci. 2005; 28(3):265-77. [PubMed: 16106155]

5. Israelski D, Gore-Felton C, Power R, Wood MJ, Koopman C. Sociodemographic characteristics associated with medical appointment adherence among HIV-seropositive patients seeking treatment in a county outpatient facility. Prev Med. 2001; 33(5):470-5. [PubMed: 11676589]

6. AIDSvu. [Available from: www.aidsvu.org.]

7. Using GTFS Data in ArcGIS Network Analyst. [Available from: http://transit.melindamorang.com/ overview_AddGTFStoND.html.]

8. Chicago Open Data Portal. [Available from: https://data.cityofchicago.org.]

9. Transitland Feed Registry. [Available from: https://transit.land/feed-registry.]

10. United States Census Bureau. [Available from: https://www.census.gov/.]

11. Health Resources and Services Administration. HIV/AIDS Bureau Performance Measures. [Available from: https://hab.hrsa.gov/sites/default/files/hab/clinical-quality-management/ coremeasures.pdf.]

12. Mugavero MJ, Davila JA, Nevin CR, Giordano TP. From access to engagement: measuring retention in outpatient HIV clinical care. AIDS Patient Care STDS. 2010; 24(10):607. [PubMed: 20858055]

13. Yehia BR, French B, Fleishman JA, Metlay JP, Berry SA, Korthuis PT, et al. Retention in care is more strongly associated with viral suppression in HIV-infected patients with lower versus higher CD4 counts. J Acquir Immune Defic Syndr. 2014; 65(3):333-9. [PubMed: 24129370]

14. Mugavero MJ, Lin HY, Willig JH, Westfall AO, Ulett KB, Routman JS, et al. Missed visits and mortality among patients establishing initial outpatient HIV treatment. Clinical infectious diseases: an official publication of the Infectious Diseases Society of America. 2009; 48(2):248-56. [PubMed: 19072715]

15. Park WB, Choe PG, Kim SH, Jo JH, Bang JH, Kim HB, et al. One-year adherence to clinic visits after highly active antiretroviral therapy: a predictor of clinical progress in HIV patients. Journal of internal medicine. 2007; 261(3):268-75. [PubMed: 17305649]

16. Eberhart MG, Voytek CD, Hillier A, Metzger DS, Blank MB, Brady KA. Travel distance to HIV medical care: a geographic analysis of weighted survey data from the Medical Monitoring Project in Philadelphia, PA. AIDS Behav. 2014; 18(4):776-82. [PubMed: 24141487]

17. Smalls BL, Gregory CM, Zoller JS, Egede LE. Assessing the relationship between neighborhood factors and diabetes related health outcomes and self-care behaviors. BMC Health Serv Res. 2015; 15:445. [PubMed: 26428459]

18. Voisin DR, Hotton A, Neilands T. Exposure to Community Violence and Sexual Behaviors Among African American Youth: Testing Multiple Pathways. Behav Med. 2016:1-9. 
19. Lucas GM, Chaisson RE, Moore RD. Highly active antiretroviral therapy in a large urban clinic: risk factors for virologic failure and adverse drug reactions. Ann Intern Med. 1999; 131(2):81-7. [PubMed: 10419445]

20. Daskalakis DC. The Electronic Health Record and Patient Portals in HIV Medicine. Camb Q Healthc Ethics. 2017; 26(2):332-6. [PubMed: 28361729]

21. Johnson MO. The shifting landscape of health care: toward a model of health care empowerment. Am J Public Health. 2011; 101(2):265-70. [PubMed: 21164096]

22. Goswami ND, Schmitz MM, Sanchez T, Dasgupta S, Sullivan P, Cooper H, et al. Understanding Local Spatial Variation Along the Care Continuum: The Potential Impact of Transportation Vulnerability on HIV Linkage to Care and Viral Suppression in High-Poverty Areas, Atlanta, Georgia. J Acquir Immune Defic Syndr. 2016; 72(1):65-72. [PubMed: 26630673] 

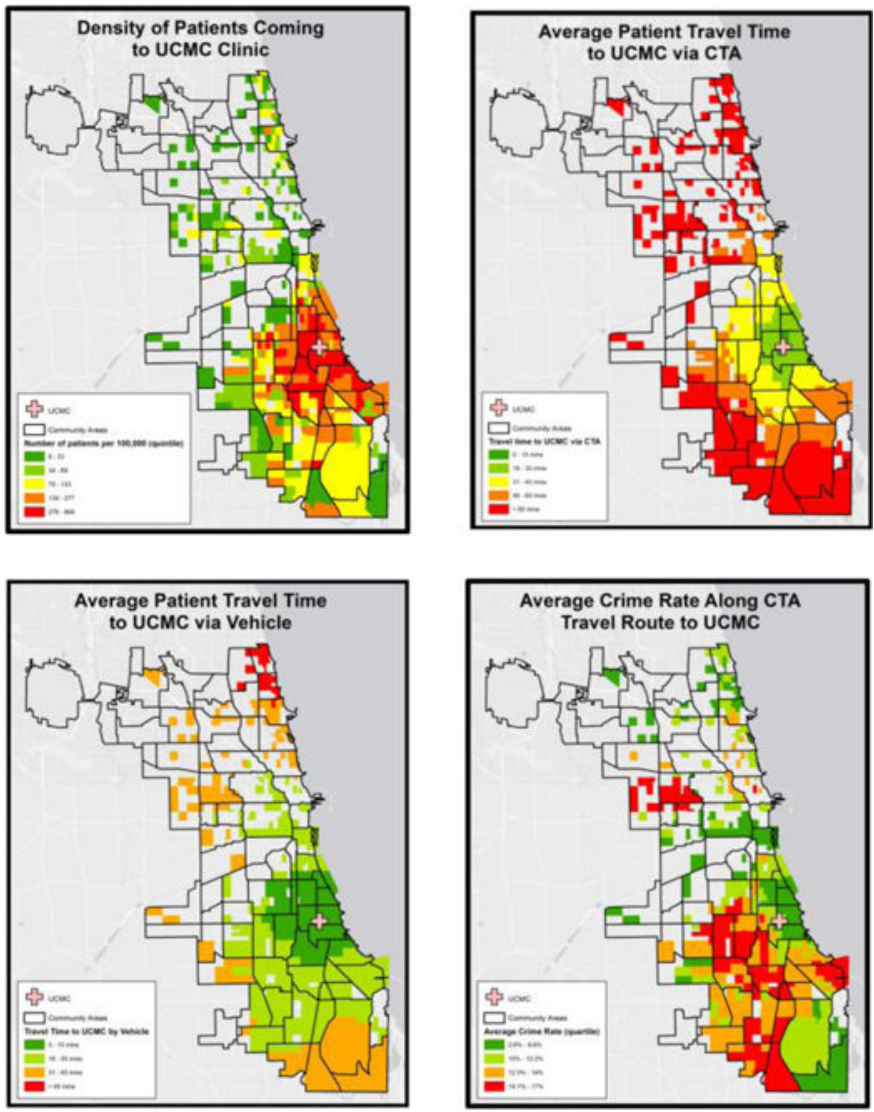

Figure 1.

Maps of patient density, travel time to clinic, and average crime rate along travel route to clinic

Abbreviations: UCMC, University of Chicago Medical Center; CTA, Chicago Transit Authority 


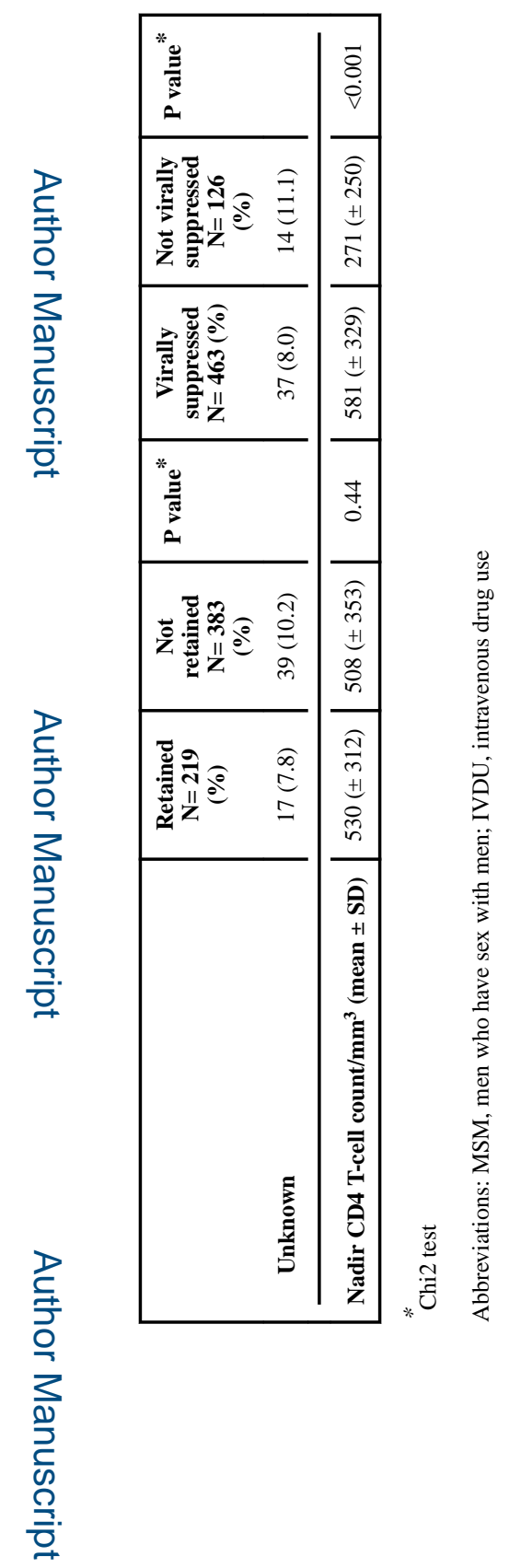

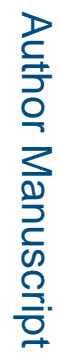




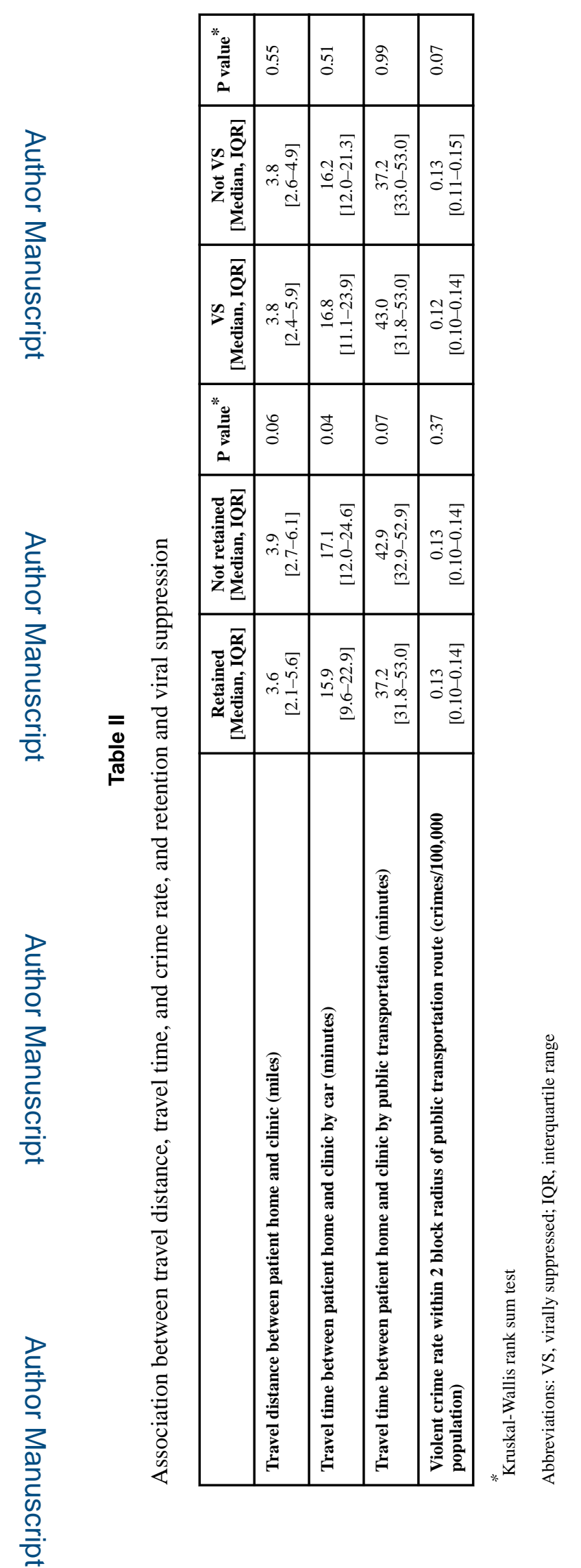

AIDS Behav. Author manuscript; available in PMC 2019 September 01. 